\title{
Variation of Isokinetic Strength and Bone Mineral Density in Youth Portuguese Soccer Players with Age
}

\author{
S. Mota ${ }^{1}$, J. Brito ${ }^{1}$, E. Passos ${ }^{1}$, E. Marques ${ }^{2}$, J. Mota $^{2}$, A. Seabra ${ }^{1}$ and A. Rebelo ${ }^{1, *}$ \\ ${ }^{I}$ Centre of Research, Education, Innovation and Intervention in Sport, Faculty of Sports, University of Porto, Portugal \\ ${ }^{2}$ Research Centre in Physical Activity, Health and Leisure, Faculty of Sports, University of Porto, Portugal
}

\begin{abstract}
The aim of this study was to study the variation of isokinetic strength and bone mineral density on the lower limb in youth soccer players according to age level. Sixty-one youth male soccer players from three age groups (Under19, Under-17, and Under-15) take part on the study. Hamstrings and quadriceps concentric strength was evaluated by isokinetic dynamometry, and body composition was assessed using Dual energy X-ray absorptiometry. The t-test of matched pairs, ANOVA, and Bonferroni post hoc comparisons were applied to localize differences by age group. Statistical criterion was set at 0.05 . Strength and bone mineral density tended to develop with age with the furthest presenting higher values in the non dominant extremity in all age groups. Differences were found in peak torque of the quadriceps in the dominant limb between Under-15 and the other age groups $(\mathrm{p}<0.05)$. For the hamstrings peak torque and bone mineral density, differences were only found between the Under-19 and Under-15 ( $\mathrm{p}<0.01$ ). The Under-15 group diverged from the other two groups in the non-dominant limb in all variables. The non-dominant limb presented higher values of bone mineral density in all three groups $(\mathrm{p}<0.001)$. Bone mineral density seems to increase due to soccer practice, and this fact seems to be connected with strength development. Increasing muscle mass, which occurs with soccer practice, may increase the forces generated at the bones, thus leading to the development of bone mineral density.
\end{abstract}

Keywords: Young athletes, football, lower limb, sports practice, health, physical activity.

\section{INTRODUCTION}

The sports practice seems to have a great developing impact during growth, both in strength [1,2] and BMD [2-6]. High impact sports, such as soccer, have an effect on the development of bone mineral density (BMD) in activities with high mechanical loads [2]. Several studies have been conducted to investigate this effect especially in women but few have been done among young soccer players [4].

The exercise takes two separate ways of developing bone resistance: one that occurs from high impact loads for sport; and an indirect way, by the development of muscles in a specific area, making a higher tension on the bone attached to them [5]. Providing attention on both strength and BMD is also paying attention on injuries. Strength adaptation on a soccer player conducts at different development in the hamstrings and quadriceps and these are the major worry because of several injuries associated to strength unbalances [7]. On the other hand, increasing BMD provides a protection against possible bone fractures [2, 4]. It is essential to understand how these adaptations occur with rising age in young soccer players. Thus, in this study we intended to analyse the variation of isokinetic muscle strength and bone mineral density on both dominant and non-dominant lower limb in Portuguese young soccer players, according to age level.

*Address correspondence to this author at the Centre of Research, Education, Innovation and Intervention in Sport, Faculty of Sports, University of Porto, Portugal; Tel: +351 225074771; Fax: +351 225500689;

E-mail: anatal@fade.up.pt

\section{METHODS}

A random sample of 71 young male soccer players was analysed and divided into 3 groups, according to age: Under$19(\mathrm{~N}=12)$, Under-17 $(\mathrm{N}=20)$ and Under-15 $(\mathrm{N}=39)$. Isokinetic dynamometry (Biodex - System II) at an angular velocity of $90^{\circ} . \mathrm{s}^{-1}$ was used to determine the maximum voluntary concentric torque of hamstring and quadriceps muscles of dominant and non-dominant lower limb. Body composition was assessed by using Dual energy X-ray absorptiometry (DEXA) Hologic Explorer ${ }^{\circledR}$ - QDR-4500, version 12.0. All subjects were evaluated with a standard applied protocol, in a supine position. Well-trained technicians made the exams and all equipments were calibrated according to the manufactures instruction. Statistical procedures were done by using SPSS 17.0 for Windows. A t-test of matched pairs was used to compare the mean values of the three variables between dominant and non-dominant lower limbs. An ANOVA analysis was used to compare the variation of the different variables between the three groups and a Bonferroni post hoc comparison was applied to localise the differences. Significance level was set at 0.05 .

\section{RESULTS}

The mean values (Mean \pm SD) of peak torque of hamstring, peak torque of the quadriceps and BMD tended to develop with age (Table 1) for all age groups. BMD also showed, higher values in the non dominant lower limb than in the dominant lower extremity. The results of t-test values showed significant differences in two variables for dominant 
Table 1. Descriptive Statistics (Mean \pm SD), T-Test and $P$ values of Matched Pairs of Peak Torque of the Quadriceps, Peak Torque of the Hamstrings and Bone Mineral Density

\begin{tabular}{|c|c|c|c|c|c|c|c|c|c|}
\hline & \multicolumn{3}{|c|}{ Dominant Lower Limb } & \multicolumn{3}{c|}{ Non Dominant Lower Limb } & \multicolumn{3}{c|}{ T-test } \\
\hline & U19 & U17 & U15 & U19 & U17 & U15 & U19 & U17 & U15 \\
\hline \hline PT $_{\mathbf{Q}}$ & $210.1 \pm 47.4$ & $176.5 \pm 28.0$ & $154.7 \pm 27.7$ & $199.1 \pm 47.6$ & $181.6 \pm 33.4$ & $152.3 \pm 34.7$ & 1.601 & 1.326 & 0.805 \\
\hline PT $_{\mathbf{H}}$ & $109.3 \pm 24.9$ & $90.1 \pm 10.2$ & $78.1 \pm 26.1$ & $101.0 \pm 30.9$ & $92 \pm 12.3$ & $70.8 \pm 21.3$ & $2.626^{\#}$ & 0.676 & $3.270^{\#}$ \\
\hline BMD & $1.48 \pm 0.15$ & $1.37 \pm 0.11$ & $1.27 \pm 0.23$ & $1.50 \pm 0.17$ & $1.41 \pm 0.11$ & $1.28 \pm 0.14$ & -1.734 & $-4.233^{*}$ & -0.551 \\
\hline
\end{tabular}

$\mathrm{PT}_{\mathrm{Q}}$, Peak torque quadriceps (N.m); $\mathrm{PT}_{\mathrm{H}}$, Peak torque Hamstring (N.m); BMD, Bone mineral density (g.cm); Differences between mean values of Dominant and Non Dominant lower limbs: ${ }^{\mathrm{p}} \mathrm{p}<0.001 ;{ }^{\#} \mathrm{p}<0.05$.

and non dominant lower limbs. In the Under-19 and Under15 groups, the peak torque of hamstrings differed from dominant to non dominant limb $(\mathrm{p}<0.05)$ and the under-17 group presented significant differences between limbs for BMD $(\mathrm{p}<0.001)$.

Significant differences were found between age groups for all the three variables analysed in both dominant and non-dominant lower limb: peak torque of hamstring $(\mathrm{F}=9.225 ; \mathrm{p}<0.001$ in the dominant $\mathrm{limb}$ and $\mathrm{F}=12.434$; $\mathrm{p}<0.001$ in the non-dominant limb), peak torque of quadriceps $(F=14.443 ; p<0.001$ in the dominant limb and $F=9.234$; $\mathrm{p}<0.001$ in the non-dominant limb), and bone mineral density $(F=6.312 ; p<0.05$ in the dominant limb; and $F=13.000$; $\mathrm{p}<0.001$ in the non-dominant limb).

In the dominant lower extremity, there were found significant differences on the peak torque of the quadriceps between Under-19 and Under-17 ( $\mathrm{p}<0.05)$ and between Under-17 and Under-15 ( $\mathrm{p}<0.001)$ groups. For the peak torque of the hamstrings and bone mineral density, there were only differences between the Under-19 and Under-15 groups ( $\mathrm{p}<0.001$ and $\mathrm{p}=0.005$, respectively). The Under-15 group presented significant differences from the other two older groups in the non-dominant lower extremity for the three variables analysed: Under-19 vs. Under-15 (peak torque of hamstring, $\mathrm{p}<0.001$; peak torque of quadriceps, $\mathrm{p}=0.001$ and BMD, $\mathrm{p}<0.001$ ); Under-17 vs. Under-15 (peak torque of hamstring, $\mathrm{p}=0.019$; peak torque of quadriceps, $\mathrm{p}=0.002$; and BMD, $p=0.005)$. Significant differences were also found between mean values of bone mineral density in both lower limbs, with the higher values found in the non-dominant lower limb, in all three groups $(\mathrm{p}<0.001)$.

\section{DISCUSSION}

The present results have shown statistical differences in the mean values for dominant and non-dominant lower limb on the peak torque of hamstrings. Under-19 and Under-15 groups presented increased strength in the hamstrings on the dominant leg as compared with non-dominant lower limb. Other studies [1] that examined the bilateral differences in young soccer players also found similar differences, concluding that young soccer players usually have higher strength values for the dominant leg.

Other important result found concerns to the difference in BMD between dominant and non-dominant lower limb: the non-preferred leg tended to have higher values than the dominant limb, but differences were only found for the Under-17. In soccer, during kicking the non-dominant lower limb supports the weight of the body which imposes a higher load to the bones of the non-dominant leg. Such biomechanical factor may lead to the higher values of BMD [5].

The development of isokinetic strength and skeletal response according to rising age in sports practice is one of the main focuses of several studies [1, 2, 4, 5-8]. However, it seems that soccer practice, requiring the repetition of several technical skills, such as kicking, jumping and landing, can promote bilateral differences between dominant and nondominant lower limbs [7], and further studies are required to understand this phenomenon [1].

The present results revealed an increase of muscle strength and BMD with the age of young soccer players and this linear increment on strength values for both peak torque of hamstring and quadriceps seems to be in accordance with other studies [5, 8], suggesting that different factors such as maturity and body size can be related with this fact. Moreover, soccer practice generates high mechanical loadings that promote the development of BMD at the loaded sites $[3,6,9$, 10]. Thus, we can speculate that older players, presenting more years of soccer practice and more exposure to the loadings, may also show higher values of BMD.

The development of strength may promote the increase of BMD [2, 4], and increasing muscle mass, which occurs with soccer practice, also increases the forces generated on the bones attached to that specific muscle, which could lead to the development of BMD. Further studies are required to understand the relationship between both the variables.

\section{REFERENCES}

[1] Kellis S, Gerodimos V, Kellis E, Manou V. Bilateral isokinetic concentric and eccentric strength profiles of knee extensors and flexors in young soccer players. Isokinet Exerc Sci 2001; 9: 319.

[2] Vicente-Rodriguez G. How does exercise affect bone development during growth? Sports Med 2006; 36: 561-9.

[3] Bellew JW, Gehrig L. A comparison of bone mineral density in adolescent female swimmers, soccer players and weight lifters. Pediatr Phys Ther 2006; 18: 19-22.

[4] Greene DA, Naughton GA, Adaptive skeletal responses to mechanical loading during adolescence. Sports Med 2006; 36: 72332.

[5] Rahnama N, Lees A, Bammaecichi E. A comparison of muscle strength and flexibility between the preferred and non-preferred leg in English soccer players. Ergonomics 2005; 48: 1568-7. 
[6] Soderman K, Bergstrom E, Lorentzon R, Alfredson H. Bone mass and muscle strength in young female soccer players. Calcif Tissue Int 2000; 67: 297-303

[7] Nichols-Richardson SM, Miller LE, Wootten DF, Ramp WK, Herbert WG. Concentric and eccentric isokinetic resistance training similarly increases muscular strength, fat-free soft tissue mass and specific bone mineral measurements in young women. Osteoporos Int 2007; 18: 789-96.

[8] Bassa E, Kotzamanidis C, Patikas D, Paraschos I. The effect of age on isokinetic concentric and eccentric moment of knee extensors. Isokinet Exerc Sci 2001; 9(4): 155-61.
[9]

Pettersson U, Alfredson H, Nordstrom P, Kenriksson-Larsén K, Lorentzon R. Bone mass in female Cross-Country skiers: Relationship between muscle strength and different BMD sites. Calcif Tissue Int 2000; 67: 199-206.

[10] Pettersson U, Nordstrom P, Alfredson H, Kenriksson Larsén K, Lorentzon R. Effect of high impact activity on bone mass and size in adolescent females: a comparative study between two different types of sports. Calcif Tissue Int 2000; 67: 207-14.

(C) Mota et al.; Licensee Bentham Open.

This is an open access article licensed under the terms of the Creative Commons Attribution Non-Commercial License

(http://creativecommons.org/licenses/by-nc/3.0/) which permits unrestricted, non-commercial use, distribution and reproduction in any medium, provided the work is properly cited. 